\title{
February 2015 Imaging Case of the Month
}

\author{
Michael B. Gotway, MD \\ Department of Radiology \\ Mayo Clinic Arizona \\ Scottsdale, AZ
}

Clinical History: A 37-year-old man, a former smoker (quit 10 years ago) presented to his physician as an outpatient with complaints of intermittent chest pain, malaise, and intermittent fever. Stress ECG and upper endoscopy were negative. His previous medical history was otherwise unremarkable. Various physicians told the patient his symptoms were due to "stress"; presumptive antibiotic treatment had no effect.

Frontal chest radiography (Figure 1) was performed.

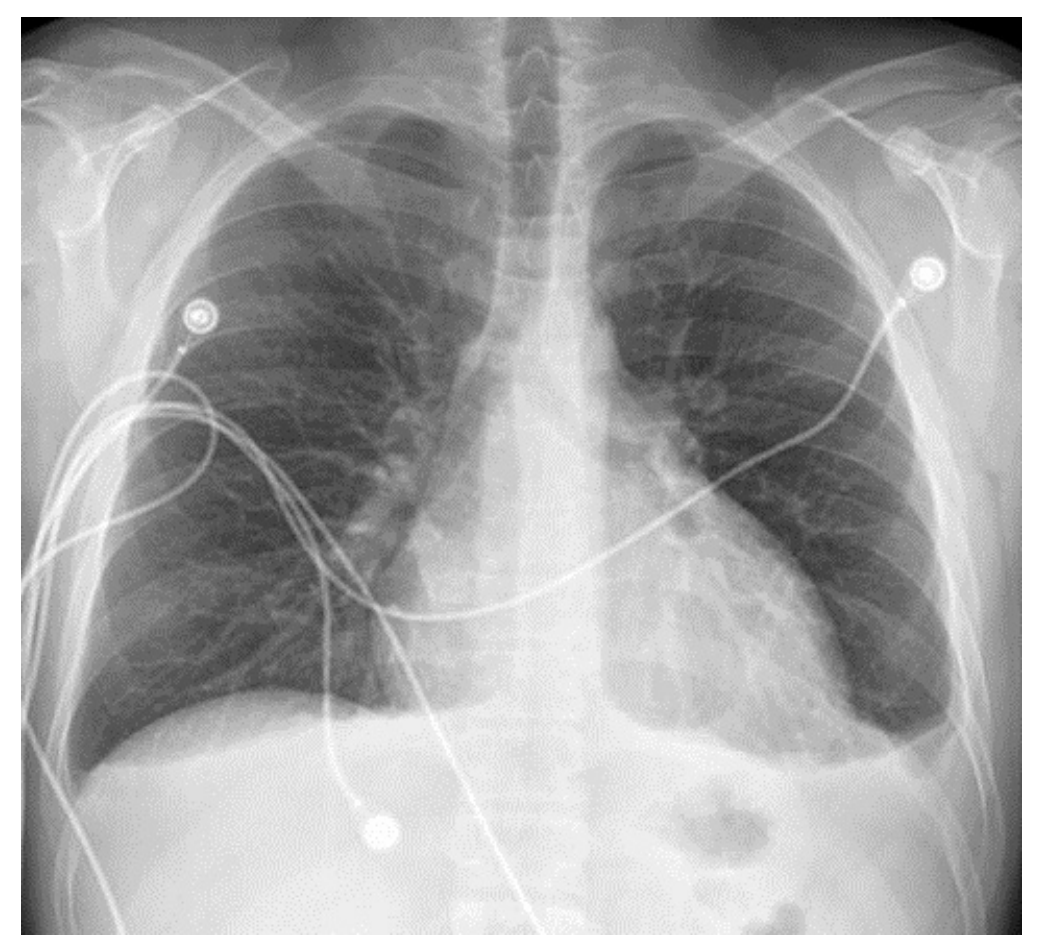

Figure 1. Frontal chest radiography.

Which of the following statements regarding the chest radiograph is most accurate?

1. The chest radiograph shows a circumscribed pulmonary mass

2. The chest radiograph shows asymmetric pulmonary vascularity

3. The chest radiograph shows bilateral linear and reticular opacities and diminished lung volumes suggesting fibrotic lung disease

4. The chest radiograph shows cardiomegaly and small pleural effusions

5. The chest radiograph shows numerous small nodules 


\section{Correct!}

\section{The chest radiograph shows cardiomegaly and small pleural effusions}

The chest radiograph shows a mildly enlarged heart with small symmetric pleural effusions. No evidence of small pulmonary nodules is seen and no mass is present. The pulmonary vascularity appears normal and symmetric, and no features to suggest fibrotic lung disease are present.

The patient's laboratory data was largely normal, aside from an elevated erythrocyte sedimentation rate of $58 \mathrm{~mm} / \mathrm{hr}$, (normal, $0-22 \mathrm{~mm} / \mathrm{hr}$ ) and a markedly elevated Creactive protein of $106.5 \mathrm{mg} / \mathrm{dL}$, (normal $<1 \mathrm{mg} / \mathrm{dL}$ ). The patient's physicians felt the patient's presentation was most suggestive of an infectious illness. The patient subsequently underwent enhanced thoracic CT (Figure 2).

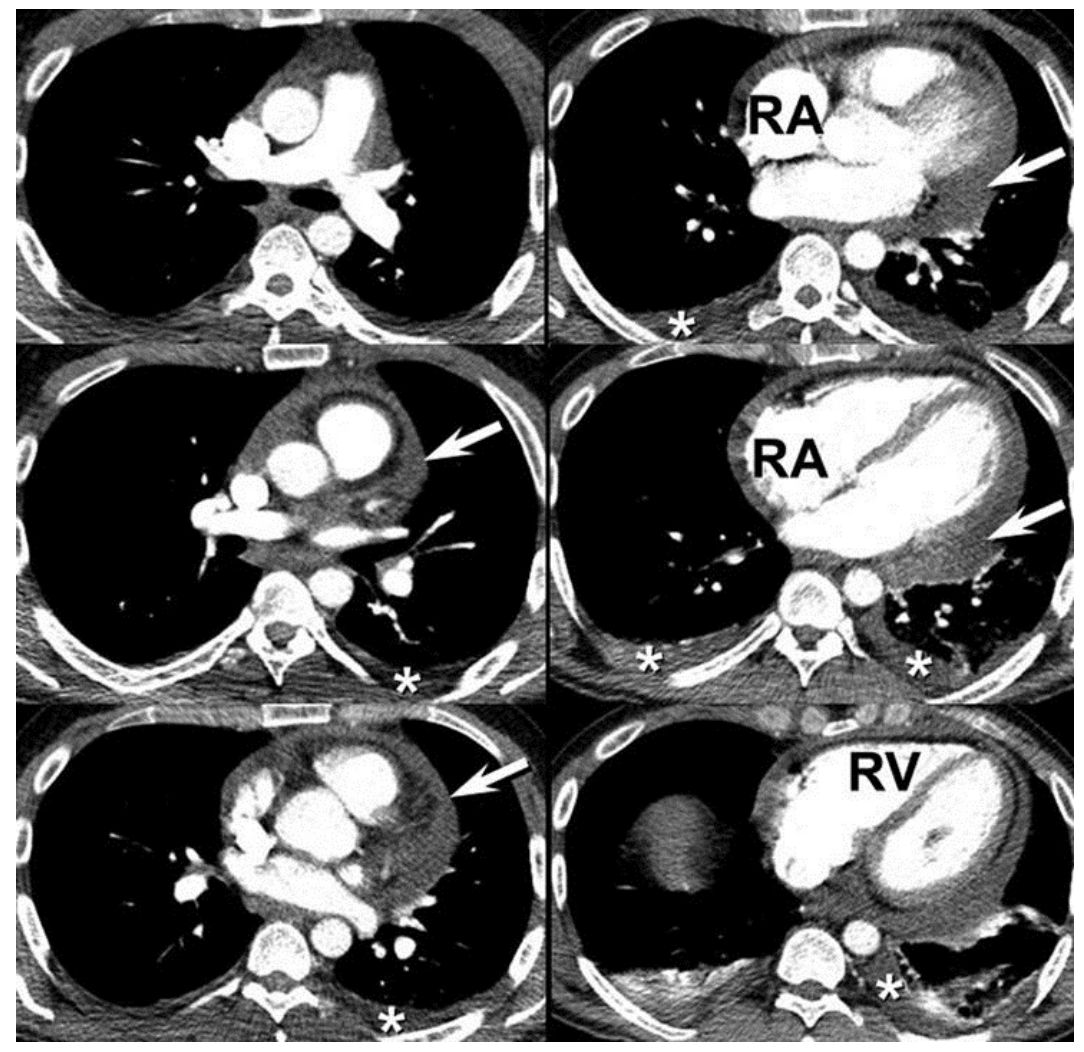

Figure 2. Representative images from the axial enhanced thoracic $\mathrm{CT}$. The right atrium (RA) and right ventricle (RV) both appear mildly enlarged

Which of the following is the regarding the thoracic CT is most accurate?

1. Thoracic CT shows a saccular thoracic aortic aneurysm

2. Thoracic CT shows bilateral pulmonary emboli

3. Thoracic CT shows diffuse soft tissue infiltration of the mediastinum

4. Thoracic CT shows lytic lesions involving the osseous structures of the thorax

5. Thoracic CT shows pleural effusions and pericardial effusions or thickening 


\section{Correct!}

\section{Thoracic CT shows pleural effusion and pericardial effusions or thickening}

The enhanced thoracic CT shows well-enhanced pulmonary arteries bilaterally- no evidence of pulmonary emboli is seen. The visualized mediastinal tissues appear normal- no evidence of abnormal infiltration or lymphadenopathy is seen. The thoracic aorta is normal in caliber. The osseous thoracic structures show no evidence of lytic or destructive processes. Small symmetric pleural effusions are evident $\left({ }^{*}\right)$, and the pericardium appears abnormal (arrows), representing either pericardial effusion or thickening, or a combination thereof.

Which of the following represents the next most appropriate step for the evaluation of this patient?

1. ${ }^{18}$ FDG-PET scanning

2. ${ }^{68} \mathrm{Ga}$ scanning

3. Catheter pulmonary angiography

4. Repeat chest radiography

5. Transthoracic echocardiography 


\section{Correct!}

\section{Transthoracic echocardiography}

The thoracic CT shows pericardial abnormalities, and, while enhanced thoracic CT provides excellent assessment of the pericardium, echocardiography can not only evaluate the morphology of the pericardium but can assess for the function consequences of pericardial abnormalities, including pericardial tamponade or pericardial constriction. Repeat chest radiography is unlikely to reveal new, additional information to that already known from the previous chest radiograph and thoracic CT. Catheter pulmonary angiography plays a limited role in modern practice in general, typically reserved for assessment and treatment of possible pulmonary emboli, treatment of arteriovenous malformations, embolization of anomalous vessels supplying sequestrations, and several other uncommon circumstances, but has little role in the evaluation of this patient. ${ }^{18} \mathrm{FDG}-\mathrm{PET}$ scanning plays a large role in the evaluation of the solitary pulmonary nodule, lung cancer staging, and the staging of extrathoracic malignancies, but has little role in the evaluation of pericardial effusions- active pericardial uptake could indicate neoplasm or inflammation of practically any cause. ${ }^{68} \mathrm{Ga}$-citrate scintigraphy is occasionally used for the assessment of diffuse lung diseases, but generally has little value in the assessment of pleural and pericardial effusions.

Transthoracic echocardiography showed a normal ejection fraction and grossly normal valvular function, and did reveal the presence of at least a small pericardial effusion However, the echocardiogram was technically challenging and was suboptimal.

Which of the following represents the next most appropriate step for the evaluation of this patient?

1. ${ }^{18}$ FDG-PET scanning

2. ${ }^{68} \mathrm{Ga}$ scanning

3. Cardiac MRI

4. Catheter pulmonary angiography

5. Transesophageal echocardiography 


\section{Correct! \\ 3. Cardiac MRI}

Transesophageal echocardiography could certainly provide excellent assessment of the heart and pericardium, but is invasive and not required in this patient. Cardiac MRI can provide excellent anatomic delineation of the cardiac structures, including assessment of valvular function, right and left ventricular ejection fractions, myocardial tissue characterization, and evaluation of the pericardium. Cardiac MRI can provide this information non-invasively, and therefore transesophageal echocardiography would not be required unless cardiac MRI could not be obtained or was technically not feasible. Catheter pulmonary angiography could provide evaluation of right heart function, which may play a role in this patient given the enlargement of the right heart seen on CT. Furthermore, right heart catheterization certainly plays a role in the evaluation of the functional consequences of pericardial disease, but obtaining catheter pulmonary angiography at this point is premature. As notes previously, both ${ }^{18} \mathrm{FDG}-\mathrm{PET}$ and ${ }^{68} \mathrm{Ga}-$ citrate scintigraphy would not prove useful for the assessment of this patient.

Cardiac MRI (Figure 3) was performed; representative images are shown below.

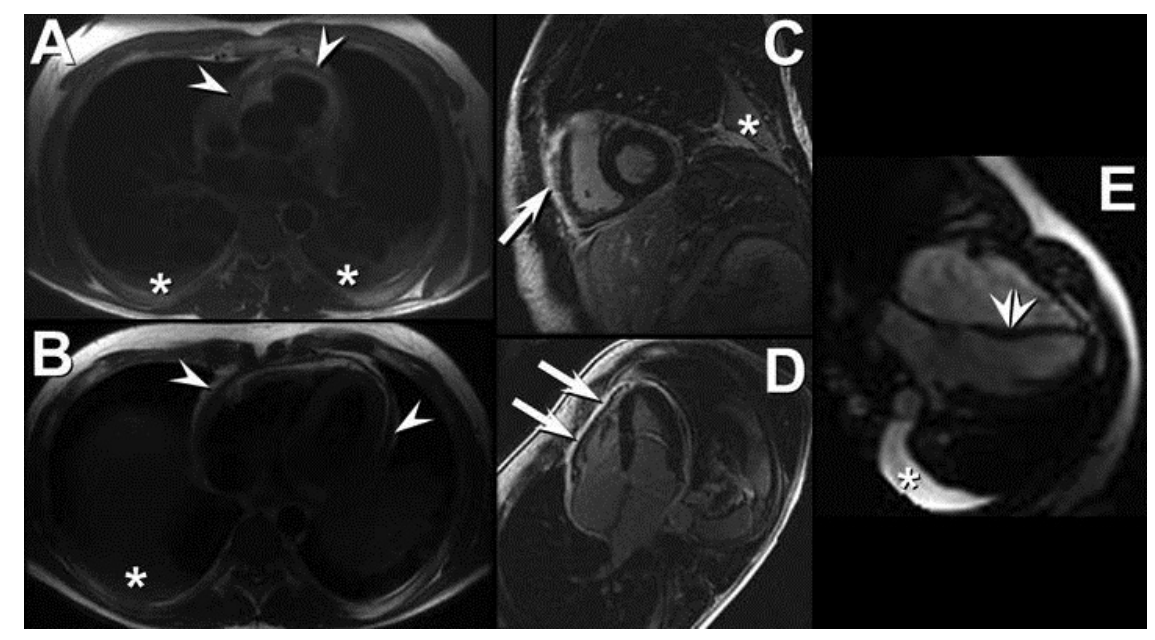

Figure 3. Axial double inversion recovery "black blood" images (A and B), short axis $(C)$ and 4 chamber (D) segmented T1-weighted inversion recovery delayed enhancement images, and free breathing MR echo $(E)$ images show pericardial thickening before intravenous contrast administration (arrowheads, A and B) with extensive pericardial thickening on delayed contrast enhanced imaging (arrows in $C$ and D). Small bilateral pleural effusions $\left(^{*}\right)$ are present. The free-breathing MR echo images show leftward motion of the interventricular septum (double arrowhead, E), representing the "septal bounce" characteristic of right ventricular pressures transiently exceeding left ventricular pressures in the setting of pericardial constriction.

The patient was treated for constrictive pericarditis with ibuprofen, prednisone, and colchicine. A repeat cardiac MRI (Figure 4) showed resolution of the pericardial thickening and enhancement, with normalization of interventricular septal motion. 


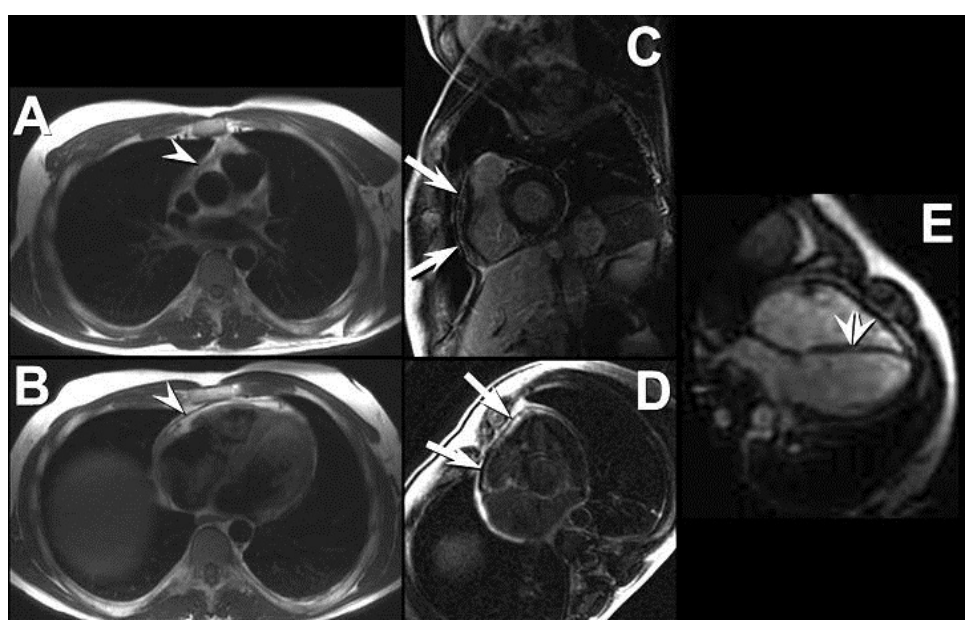

Figure 4: Axial double inversion recovery "black blood" images (A and $B$ ), short axis $(C)$ and 4 chamber (D) segmented T1-weighted inversion recovery delayed enhancement images, and free breathing MR echo (E) images show resolution of the previously thickened pericardium (arrowheads in A and B show the normal pericardium now appearing as a thin, black line). The previously seen extensive pericardial enhancement has resolved (the arrows in $C$ and $D$ show the thin black line representing the pericardium, outlined on either side by bright, hyperintense fat. The functional MR echo images show normalization of the interventricular septal position, now bowing rightward

(double arrowheads, E). Note resolution of the previously seen bilateral pleural effusions.

The patients symptoms resolved and his inflammatory biomarkers returned to normal levels as well. Repeat chest radiography (Figure 5) showed normalization of heart size and resolution of pleural effusions.

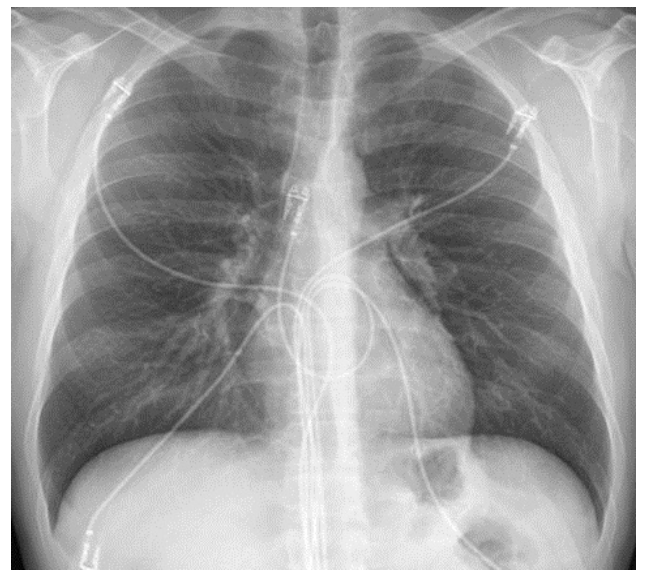

Figure 5: Frontal chest radiograph performed following treatment for constrictive pericarditis shows normalization of heart size and resolution of bilateral pleural effusions.

Diagnosis: Constrictive pericarditis 


\section{References}

1. Angheloiu GO, Rayarao G, Williams R, Yamrozik J, Doyle M, Biederman RW. Magnetic resonance characterization of septal bounce: findings of blood impact physiology. Int J Cardiovasc Imaging. 2015;31(1):105-13. [CrossRef] [PubMed]

2. Goenka AH, Wang H, Flamm SD. Cardiac magnetic resonance imaging for the investigation of cardiovascular disorders. Part 2: emerging applications. Tex Heart Inst. 2014; 41(2):135-43. [CrossRef] [PubMed]

3. Azarisman SM, Richardson JD, Chua SK, Cunningham MS, Teo KS, Worthley SG. An ideal image: effusive constrictive pericarditis. Am J Med. 2013;126(1):25-26. [CrossRef] [PubMed]

4. Peebles CR, Shambrook JS, Harden SP. Pericardial disease--anatomy and function. Br J Radiol. 2011;84 Spec No 3:S324-337. [CrossRef] [PubMed]

5. Thavendiranathan P, Verhaert D, Walls MC, Bender JA, Rajagopalan S, Chung YC, Simonetti OP, Raman SV. Simultaneous right and left heart real-time,free-breathing CMR flow quantification identifies constrictive physiology. JACC Cardiovasc Imaging. 2012;5(1):15-24. [CrossRef] [PubMed]

6. Walker CM, Chung JH, Reddy GP. "Septal bounce". J Thorac Imaging. 2012;27(1):W1. [CrossRef] [PubMed]

7. Srichai MB. CMR imaging in constrictive pericarditis: is seeing believing? JACC Cardiovasc Imaging. 2011;4(11):1192-1194 [CrossRef] [PubMed]

8. Miller CA, Dormand H, Clark D, Jones M, Bishop P, Schmitt M. Comprehensive characterization of constrictive pericarditis using multiparametric CMR. JACC Cardiovasc Imaging. 2011;4(8):917-920. [CrossRef] [PubMed]

9. Bogabathina $\mathrm{H}$, Biederman RW. Lack of slippage by cardiovascular magnetic resonance imaging is sine qua non for constrictive pericarditis. Circulation. 2011;123(16):e418-419. [CrossRef] [PubMed] 